\title{
REVISORES 2016
}

La Revista Enfermería Herediana agradece la colaboración de los expertos nacionales e internacionales que colaboraron de modo desinteresado en la revisión de los artículos científicos recibidos.

En el año 2017 contaremos con profesionales destacados y expertos en sus respectivas áreas quienes contribuirán con sus aportes a elevar la calidad de nuestra Revista Enfermería Herediana.

Mg. Clara Carmen Torres Deza

Mg. Maria Diana Ruvalcaba Rodriguez

Dra. Elvira Mercedes Sánchez Díaz

Lic. Esp. Eva Veronica Chanamé Ampuero

Dr. Carlos Tello Pompa

Dra. Luz Aurora Carbajal Arroyo

Mg. Margarita Alayo Sarmiento

Dr. Milton Carlos Guevara Valtier

Mg. Patricia Soledad Obando Castro

Mg. Margarita Cárdenas Giménez

Mg Carolina Cerna Silva
Universidad Peruana Cayetano Heredia - Perú

Universidad Autónoma de Nuevo León Monterrey - México

Universidad Peruana Cayetano Heredia - Perú

Universidad Peruana Cayetano Heredia - Perú

Universidad Nacional de Trujillo - Perú

Universidad Peruana Cayetano Heredia - Perú

Universidad Peruana Cayetano Heredia - Perú

Universidad Autónoma de Nuevo León Monterrey - México

Universidad Peruana Cayetano Heredia - Perú

Universidad Autónoma - México

Universidad Peruana Cayetano Heredia - Perú 